\title{
Uncatalyzed Synthesis of Heteroarylidine Derivatives in Aqueous Medium and their Antibacterial Evaluation
}

\author{
PRADEEP K. PALIWAL, SRINIVASA RAO JETTI and SHUBHA JAIN* \\ Laboratory of Heterocycles, School of Studies in Chemistry \& Biochemistry, \\ Vikram University, Ujjain-456010, Madhya Pradesh, India \\ drshubhajain479@gmail.com
}

Received 28 May 2012 / Accepted 15 June 2012

\begin{abstract}
Knoevengal condensation of heteroaryl aldehydes with active methylene compounds in aqueous medium at room temperature is described. The products are obtained with excellent yields in short reaction times without using any catalyst. Products require no further purification. Newly synthesized compounds have shown good to excellent biological activities against Staphylcoccus aureus, Klebsialla pneumoniae, Escherichia coli and Pseudomonas aeruginosa.
\end{abstract}

Keywords: Knoevenagel condensation, Aqueous media, Antibacterial activity, Heteroarylidine

\section{Introduction}

The synthesis of electrophilic olefins from active methylene and carbonyl compounds, known as the Knoevenagel condensation ${ }^{1}$. Knoevenagel condensation is one of the most well known reactions in organic chemistry owing to its applicability in the preparation of various synthetic targets ${ }^{2}$. Many modifications have been made to this process in recent years using Lewis acid catalysis ${ }^{3}$, ionic liquids ${ }^{4}$, microwave irradiation ${ }^{5}$, quaternary ammonium salts $^{6}$, heterogeneous catalysts ${ }^{7}$, organo-base mediation ${ }^{8}$, zeolites ${ }^{9}$ and in aqueous media ${ }^{10}$.

However, in many of these methods relatively harsh conditions are required, expensive reagents are involved, or a combination of several additives is employed. By keeping all these things in mind, we decided to search a new way to synthesize Knoevengal condensed products through ecofriendly, economically favorable conditions. We report here, a very simple and highly efficient method for the condensation of various 5-membered heteroaryl aldehydes with several active methylene compounds like malononitrile, ethyl cyanoacetate, and dimedone in water at room temperature. It was stimulating to observe that all the reactions occurred rapidly and were complete in a few minutes giving excellent yields of the Knoevenagel products. 


\section{Experimental}

All the melting points reported are uncorrected and were taken in open capillaries. The IR spectra were recorded on Shimadzu FT-IR spectrometer using $\mathrm{KBr}\left(\mathrm{v} \mathrm{cm}^{-1}\right)$. The ${ }^{1} \mathrm{H}-\mathrm{NMR}$ spectra were taken on Bruker DRx-600 spectrometer using TMS (Tetra methyl silane) as internal standard and $\mathrm{CDCl}_{3}$ as solvent. All chemical shift values were recorded as $\delta \mathrm{ppm}$. Mass spectra (FAB) were recorded on Jeol Sx-600 mass spectrometer using $m$-nitro benzyl alcohol as matrix. The purity of compounds was checked by TLC using silica gel-G adsorbent. All solvents and reagents were purchased from Aldrich and Merck with highgrade quality and used without any purification.

\section{General procedure for the synthesis of heteroarylidine derivatives $3(a-r)$}

To a stirred solution of activemethylene compound 2a $(10 \mathrm{mmol})$ in water $(10 \mathrm{~mL})$ was added heteroaryl aldehyde (1a) in equimolar ratio, rapidly and all at once. The progress of the reaction was monitored by TLC. After few minutes, the solid produced was isolated by simple filtration and dried. The solid product 3a (98\%) was identified by spectroscopic measurements and by comparison with an authentic samples, needed no further purification. Similarly, utilizing the aldehydes $\mathbf{1}(\mathbf{a}-\mathbf{f})$ and activemethylene compounds $\mathbf{2 ( a - c )}$, compounds 3(a-r) were synthesized and characterized (Table 1).

Table 1. Synthesis of heteroarylidine derivatives 3(a-r) in aqueous media

\begin{tabular}{|c|c|c|c|c|c|c|c|}
\hline \multirow{2}{*}{ Entry } & \multirow{2}{*}{ Aldehyde } & \multirow{2}{*}{$\mathrm{AMC}^{*}$} & \multirow{2}{*}{ Product $^{\mathrm{a}}$} & \multirow{2}{*}{$\begin{array}{c}\text { Time, } \\
\text { min }\end{array}$} & \multirow{2}{*}{$\begin{array}{c}\text { Yield, } \\
\%^{\mathrm{b}}\end{array}$} & \multicolumn{2}{|c|}{ M.P., ${ }^{0} \mathrm{C}$} \\
\hline & & & & & & Found & Reported \\
\hline 1 & $1 \mathrm{a}$ & $2 a$ & $3 a$ & 8 & 98 & 120 & $120-12^{2 \mathrm{a}}$ \\
\hline 2 & $1 b$ & $2 \mathrm{a}$ & $3 b$ & 10 & 96 & 92 & $91-92^{7 b}$ \\
\hline 3 & $1 \mathrm{c}$ & $2 a$ & $3 c$ & 12 & 93 & 72 & $73^{2 \mathrm{a}}$ \\
\hline 4 & $1 d$ & $2 a$ & $3 d$ & 13 & 95 & 98 & $98-99^{7 b}$ \\
\hline 5 & $1 \mathrm{e}$ & $2 a$ & $3 e$ & 8 & 92 & 96 & 97-99 \\
\hline 6 & 1f & $2 a$ & $3 f$ & 9 & 95 & 101 & $101-102^{7 b}$ \\
\hline 7 & $1 \mathrm{a}$ & $2 b$ & $3 g$ & 8 & 96 & $135-136$ & $135-137^{7 b}$ \\
\hline 8 & $1 b$ & $2 b$ & $3 \mathrm{~h}$ & 7 & 98 & $105-106$ & $105-108^{7 b}$ \\
\hline 9 & $1 \mathrm{c}$ & $2 b$ & $3 \mathrm{i}$ & 9 & 97 & 93 & $94^{2 \mathrm{a}}$ \\
\hline 10 & $1 d$ & $2 b$ & $3 \mathrm{j}$ & 9 & 98 & 70 & - \\
\hline 11 & $1 \mathrm{e}$ & $2 b$ & $3 \mathrm{k}$ & 8 & 98 & 88 & - \\
\hline 12 & $1 f$ & $2 b$ & 31 & 9 & 90 & 104 & - \\
\hline 13 & $1 \mathrm{a}$ & $2 c$ & $3 m$ & 10 & 96 & 154 & 156 \\
\hline 14 & $1 b$ & $2 c$ & $3 n$ & 8 & 95 & 140 & $140-142$ \\
\hline 15 & $1 \mathrm{c}$ & $2 c$ & 30 & 10 & 93 & 158 & $158-159$ \\
\hline 16 & $1 d$ & $2 c$ & $3 p$ & 12 & 90 & 152 & - \\
\hline 17 & $1 \mathrm{e}$ & $2 c$ & $3 q$ & 12 & 91 & 146 & - \\
\hline 18 & $1 \mathrm{f}$ & $2 c$ & $3 r$ & 11 & 92 & 156 & - \\
\hline
\end{tabular}

\section{Antibacterial evaluation}

The bacterial species employed were strains of Staphylococcus aureus, Klebsialla pneumoniae, Escherichia coli and Pseudomonas aeruginosa. The isolates were biochemically tested $^{11,12}$. The strains were maintained on trypticase soya agar (TSA). The bacterial inoculam were prepared from $20 \mathrm{~mL}$ of overnight stock culture in tryptone soya 
broth (TSB) at $37 \pm 2{ }^{\circ} \mathrm{C}$. The Muller Hinton agar (MHA) was used for sensitivity test. The compounds were tested at $2 \mathrm{mg} / \mathrm{mL}$ concentration in ethanol against all organisms. Inhibition was recorded by measuring the diameter of the inhibition zone at the end of $24 \mathrm{~h}$ for bacteria at $37 \pm 2{ }^{\circ} \mathrm{C}$. All isolates of selected strains show sensitivity against synthesized molecules. The antibacterial susceptibility test of the selected strains was determined by standard disc diffusion method ${ }^{13,14}$. Each experiment was repeated thrice and the average of the three independent determinations was recorded. The protocols were summarized in Table 2.

Table 2. Antibacterial activities heteroarylidine derivatives (Zone of inhibition in $\mathrm{mm}$ )

\begin{tabular}{cccccc}
\hline Entry & Product & S. aureus & K. pneumoniae & E. coli & P. aeruginosa \\
\hline 1 & $3 \mathrm{a}$ & + & +++ & ++ & + \\
2 & $3 \mathrm{~b}$ & +++ & + & + & +++ \\
3 & $3 \mathrm{c}$ & + & + & + & + \\
4 & $3 \mathrm{~d}$ & + & ++ & + & ++ \\
5 & $3 \mathrm{e}$ & ++ & + & ++ & + \\
6 & $3 \mathrm{f}$ & ++ & + & + & + \\
7 & $3 \mathrm{~g}$ & + & +++ & +++ & ++ \\
8 & $3 \mathrm{~h}$ & +++ & + & + & +++ \\
9 & $3 \mathrm{i}$ & + & + & + & + \\
10 & $3 \mathrm{j}$ & + & ++ & + & +++ \\
11 & $3 \mathrm{k}$ & +++ & + & + & + \\
12 & 31 & + & + & + & + \\
13 & $3 \mathrm{~m}$ & + & +++ & ++ & ++ \\
14 & $3 \mathrm{n}$ & ++ & ++ & +++ & + \\
15 & $3 \mathrm{o}$ & + & + & ++ & ++ \\
16 & $3 \mathrm{p}$ & + & + & + & + \\
17 & $3 \mathrm{q}$ & ++ & ++ & ++ & ++ \\
18 & $3 \mathrm{r}$ & + & ++ & ++ & + \\
\hline
\end{tabular}

Zone area $+=1-5 \mathrm{~mm},++=6-10 \mathrm{~mm},+++=11-15 \mathrm{~mm}$

\section{Selected Spectral data}

\section{2-(1H-2-pyrrolylmethylene) malononitrile (3a)}

Light gray solid, m.p. 120-122 ${ }^{\circ} \mathrm{C}$, IR (KBr): 3368, 2998, 2226, 589, 1511, 1393, 1322, 1123, 1045, 927, 868, 769, 700, 583. ${ }^{1} \mathrm{H}$ NMR $\left(200 \mathrm{MHz}, \mathrm{CDCl}_{3}\right) \delta: 6.40(\mathrm{dd}$, $\mathrm{J}=2.5,3.4 \mathrm{~Hz}, 1 \mathrm{H}), 7.20(\mathrm{~d}, \mathrm{~J}=2.5 \mathrm{~Hz}, 1 \mathrm{H}), 7.40(\mathrm{~d}, \mathrm{~J}=3.4 \mathrm{~Hz}, 1 \mathrm{H}), 7.65(\mathrm{~s}, 1 \mathrm{H})$, 11.5(br. s, $1 \mathrm{H} \mathrm{NH})$ ppm. ${ }^{13} \mathrm{C} \mathrm{NMR}\left(\mathrm{CDCl}_{3}, 75 \mathrm{MHz}\right): \delta=69.5,113.2,114.5,115.5$, 125.2, 126.6, 130.2, 149.9 ppm. EI-MS: $m / z(\%)=143(100)[\mathrm{M}+], 116(25), 92(67), 39$ (24), 28(38), $\mathrm{C}_{8} \mathrm{H}_{5} \mathrm{~N}_{3}$ (143.15): calcd. C 67.13, H 3.52, N 29.35 found $\mathrm{C} 67.19, \mathrm{H}$ $3.45, \mathrm{~N} 29.41$.

\section{2-(2-Furylmethylene) malononitrile (3c)}

Pale yellow solid, m.p. 67-68 ${ }^{\circ} \mathrm{C}$, IR (KBr) : 3415, 3041, 2221, 1596, 1525, 1456, 1391, 1299, 1149, 1024, 923, 765, $582{ }^{1} \mathrm{H}$ NMR $\left(200 \mathrm{MHz}, \mathrm{CDCl}_{3}\right) \delta: 6.75(\mathrm{dd}, \mathrm{J}=3.9,1.8 \mathrm{~Hz}$, $1 \mathrm{H}), 7.40(\mathrm{~d}, \mathrm{~J}=3.9 \mathrm{~Hz}, 1 \mathrm{H}), 7.55(\mathrm{~d}, \mathrm{~J}=1.8 \mathrm{~Hz}, 1 \mathrm{H}), 7.8(\mathrm{~s}, 1 \mathrm{H}) \mathrm{ppm} .{ }^{13} \mathrm{C} \mathrm{NMR}\left(\mathrm{CDCl}_{3}, 75\right.$ MHz): $\delta=77.2,112.5,113.7,114.3,123.5,143.0,147.9,149.5 \mathrm{ppm}$. EI-MS: $\mathrm{m} / z(\%)=$ 144(100) [M+], 141(90), 115(35), 89 (20), 69(14), 62(13), 43(18). $\mathrm{C}_{8} \mathrm{H}_{4} \mathrm{~N}_{2} \mathrm{O}$ (144.13): calcd. C 66.67, H 2.80, N 19.44 found C 66.62, H 2.84, N 19.51. 


\section{Ethyl (E)-2-cyano-3-(2-thienyl)-2-propionate (3h)}

Pale yellow solid, m.p. 105-108 ${ }^{\circ} \mathrm{C}$, IR (KBr): 3083, 2223, 1717, 1608, 1465, 1334, 1244 , 1207, 1083, 1005, 758. ${ }^{1} \mathrm{H}$ NMR $\left(200 \mathrm{MHz}, \mathrm{CDCl}_{3}\right) \delta: 1.40(\mathrm{t}, \mathrm{J}=6.9 \mathrm{~Hz}, 3 \mathrm{H}), 4.38$ $(\mathrm{q}, \mathrm{J}=6.9 \mathrm{~Hz}, 2 \mathrm{H}), 7.20-7.28(\mathrm{~m}, \mathrm{~J}=1 \mathrm{H}), 7.78(\mathrm{~d}, \mathrm{~J}=4.5 \mathrm{~Hz}, 1 \mathrm{H}), 7.85(\mathrm{~d}, \mathrm{~J}=2.1 \mathrm{~Hz}, 1 \mathrm{H})$, 8.30(s, 1H) ppm. ${ }^{13} \mathrm{C} \mathrm{NMR}\left(\mathrm{CDCl}_{3}, 75 \mathrm{MHz}\right): \delta=14.1,62.4,99.2,115.6,128.5,130.0$, 135.9, 137.1, 146.6, 162.5 ppm. EI-MS: $m / z(\%)=207(100)[\mathrm{M}+], 179(52), 162(95), 134$ (72), 108(34), 90 (30), 45(35). $\mathrm{C}_{10} \mathrm{H}_{9} \mathrm{NO}_{2} \mathrm{~S}$ (207.25): calcd. C 58.12, H 4.38, N 6.76, S 15.47 found C 58.14, H 4.41, N 6.86, S 15.43.

\section{Ethyl (E)-2-cyano-3-(2-furyl-2-propionate (3i)}

Coloureless solid, m.p. 89-91 ${ }^{\circ} \mathrm{C}$, IR (KBr) : 3034, 2216, 1760, 1618, 1530, 1462, 1382, 1217, 1091, 923, 758. ${ }^{1} \mathrm{H}$ NMR $\left(200 \mathrm{MHz}, \mathrm{CDCl}_{3}\right) \delta: 1.38(\mathrm{t}, \mathrm{J}=7.1 \mathrm{~Hz}, 3 \mathrm{H}), 4.35$ $(\mathrm{q}, \mathrm{J}=7.1 \mathrm{~Hz}, 2 \mathrm{H}), 6.65(\mathrm{dd}, \mathrm{J}=1.9,4.0 \mathrm{~Hz}, 1 \mathrm{H}), 7.40(\mathrm{~d}, \mathrm{~J}=4.0 \mathrm{~Hz}, 1 \mathrm{H}), 7.55(\mathrm{~d}, \mathrm{~J}=1.9$ $\mathrm{Hz}, 1 \mathrm{H}), 8.0(\mathrm{~s}, 1 \mathrm{H}) \mathrm{ppm} .{ }^{13} \mathrm{C} \mathrm{NMR}\left(\mathrm{CDCl}_{3}, 75 \mathrm{MHz}\right): \delta=13.9,62.3,98.3,113.7,115.1$, 121.6, 139.2, 148.1, 148.5, $162.3 \mathrm{ppm}$. EI-MS: $m / z(\%)=191(100)[\mathrm{M}+], 163(18)$, 146(14), 63 (20). $\mathrm{C}_{10} \mathrm{H}_{9} \mathrm{NO}_{3}$ (191.18): calcd. C 62.82, $\mathrm{H} 4.74, \mathrm{~N} 7.33$ found $\mathrm{C} 62.87, \mathrm{H}$ $4.81, \mathrm{~N} 7.42$.

\section{5,5-Dimethyl-2-((5-methylfuran-2-yl)methylene)cyclohexane-1,3-dione (3p)}

Light yellow solid, $152-156{ }^{\circ} \mathrm{C}$, IR (KBr): 3083, 1745, 1608, 1465, 1244, 1207, 1083, 1005, 758. ${ }^{1} \mathrm{H}$ NMR $\left(200 \mathrm{MHz}, \mathrm{CDCl}_{3}\right) \delta: 1.11(\mathrm{~s}, 6 \mathrm{H}), 2.29(\mathrm{~s}, 4 \mathrm{H}), 7.21-7.27(\mathrm{~m}, \mathrm{~J}=1 \mathrm{H}), 7.72(\mathrm{~d}$, $\mathrm{J}=4.6 \mathrm{~Hz}, 1 \mathrm{H}), 7.83(\mathrm{~d}, \mathrm{~J}=2.0 \mathrm{~Hz}, 1 \mathrm{H}), 8.21(\mathrm{~s}, 1 \mathrm{H}) \mathrm{ppm} .{ }^{13} \mathrm{C} \mathrm{NMR}\left(\mathrm{CDCl}_{3}, 75 \mathrm{MHz}\right): \delta$ $=14.3,26.8,30.0,52.3,109.6,112.4,145.2,146.4,149.7,154.8,185.4$ ppm. EI-MS: $\mathrm{m} / \mathrm{z}$ $(\%)=232[\mathrm{M}+], 217(100), 201(95), 150(72), 81(34) . \mathrm{C}_{14} \mathrm{H}_{16} \mathrm{O}_{3}$ (232.27): calcd. $\mathrm{C}=72.39$, $\mathrm{H}=6.94$. Found $\mathrm{C}=72.35, \mathrm{H}=6.96$.

\section{5,5-Dimethyl-2-[(5-methyl-2-thienyl)methylene]cyclohexane-1,3-dione (3q)}

Yellow solid, $140{ }^{\circ} \mathrm{C}$, IR (KBr): 3055, 1738, 1608, 1465, 1244, 1207, 1083, 1005, 758. ${ }^{1} \mathrm{H}$ NMR $\left(200 \mathrm{MHz}, \mathrm{CDCl}_{3}\right) \delta: 1.10(\mathrm{~s}, 6 \mathrm{H}), 2.27(\mathrm{~s}, 4 \mathrm{H}), 7.24-7.30(\mathrm{~m}, \mathrm{~J}=1 \mathrm{H}), 7.78(\mathrm{~d}$, $\mathrm{J}=4.4 \mathrm{~Hz}, 1 \mathrm{H}), 7.84(\mathrm{~d}, \mathrm{~J}=2.3 \mathrm{~Hz}, 1 \mathrm{H}), 8.24(\mathrm{~s}, 1 \mathrm{H}), 2.34(\mathrm{~s}, 3 \mathrm{H}) \mathrm{ppm} .{ }^{13} \mathrm{C} \mathrm{NMR}$ $\left(\mathrm{CDCl}_{3}, 75 \mathrm{MHz}\right): \delta=14.8,26.9,30.4,52.6,127.9,130.4,135.9,142.9,145.5,148.2$, 149.7, $184.8 \mathrm{ppm}$. EI-MS: $\mathrm{m} / z(\%)=248(100)[\mathrm{M}+], 203(52), 178(46) 162(95), 123$ (72). $\mathrm{C}_{14} \mathrm{H}_{16} \mathrm{O}_{2} \mathrm{~S}$ (248.34): calcd. $\mathrm{C}=67.71, \mathrm{H}=6.49, \mathrm{~S}=12.91$ found $\mathrm{C}=67.71, \mathrm{H}=6.49$, $\mathrm{S}=12.95$.

\section{Results and Discussion}

In this report, we wish to highlight our findings on uncatalyzed condensation of heteroaryl aldehyde with activemethylene compounds and their biological activities. Water itself has been employed as an efficient catalyst for this reaction. In all the cases, the reaction proceeds smoothly with same reaction conditions. The reaction is highly stereoselective affording $\alpha, \beta$ - unsaturated compounds in excellent yields with an $E$-geometry. Furthermore, the treatment of aldehydes with malononitrile and dimedone also gave trisubstituted olefinic products under similar reaction conditions. The use of 5-membered heterocyclic aldehydes and their derivatives 1(a-f) gave comparatively higher yields than previously reported aliphatic or aryl aldehydes (Scheme 1). In general, the reactions are clean and free from the formation of side products. In addition the use of water as a medium helps to avoid the use of environmentally unfavorable organic solvents. 


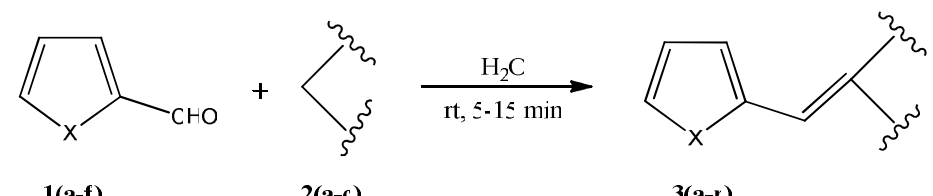<smiles>Cc1ccc(C=O)o1</smiles>

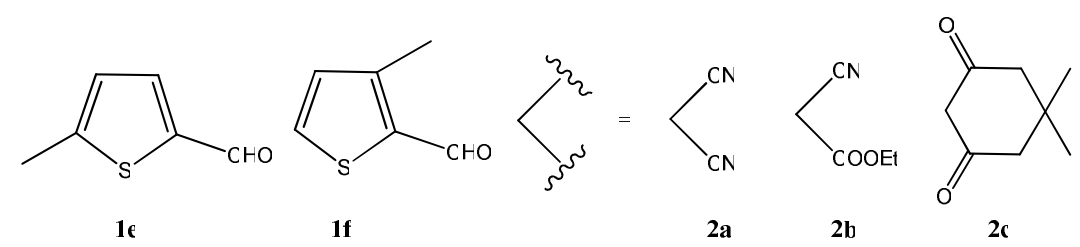

\section{Scheme 1}

From the screening results, molecules $\mathbf{3 h}, \mathbf{3 b}$ and $\mathbf{3 k}$ displayed broad-spectrum antimicrobial activity against $S$. aureus, $\mathbf{3 a}, \mathbf{3 g}$ and $\mathbf{3 m}$ against $K$. pneumoniae, $\mathbf{3 g}$ and $\mathbf{3 n}$ against $E$. coli and $\mathbf{3 b}, \mathbf{3 h}$ and $\mathbf{3 j}$ against $P$. aeruginosa, remaining products shown moderate activity.

\section{Conclusion}

In conclusion, we have demonstrated a very simple and highly efficient method for the condensation of heteroaromatic aldehydes with various activemethylene compounds to give Knoevenagel products in excellent yields. The attractive features of this procedure are operational simplicity and cleaner reaction profile. Replacement of organic solvents by water is the desirable factor for the preparation of trisubstituted olefins. Reaction completes in few minutes and no further purification is required to obtain pure product. The products obtained are suitable starting materials for the synthesis of densely substituted molecules of pharmaceutical interest. The further work is in progress on Knoevenagel condensed products in our laboratory. We suggest that the present method may displace all other methods that use various organic solvents, catalysts, MW irradiation and that are performed at high temperatures.

\section{Acknowledgement}

Authors are thankful to Deputy Director and Head, SAIF, Central Drug Research Institute (CDRI), Lucknow for providing spectral data, Department of Chemistry, Vikram University, Ujjain for extending laboratory facilities and IR data. The authors are also thankful to Mr. Kapilesh Jadhav, Lok Manya Tilak Sc. \& Comm. College, Ujjain for providing microbiological facilities

\section{References}

1. Jones G, Org React, John Wiley \& Sons: New York, 1967, 15, 204.

2. (a) Wang J, Discordia R P, Crispino G A, Li J, Grosso J A, Polniaszek R and Truc V C, Tetrahedron Lett., 2003, 44, 4271; (b) Sabitha G, Reddy G S K, Rajkumar K M, Yadav J S, Ramakrishna K V S and Kunwar A C, Tetrahedron Lett., 2003, 44(40), 7455-7457. 
3. Cabello J A, Campelo J M, Angel G, Diego L and Jose M M, J Org Chem., 1984, 49, 5195.

4. (a) Guo-hua G A O, Liang L U, Ting Z O U, Jin-bao G A O, Ye L I U and Ming-yuan H E, Chemical Research in Chinese Universities, 2007, 23(2), 169-172; (b) Reddy B M, Patil M K, Rao K N and Reddy G K, J Mole Cat A: Chem., 2006, 258(1-2), 302-307.

5. Mogilaiah K, Sharath B H, Vidya K and Kumar K S, Indian J Chem., 2010, 49B, 390.

6. Shahrzad A and Saeed B, Tetrahedron Lett., 2007, 48(18), 3299-3303.

7. (a) Seong-Jin H, Mi-Hye L and Chang-Hee L, Bull Korean Chem Soc., 2004, 25(10), 1545-1550; (b) Yadav J S, Reddy B S, Basak A K, Visali B, Narsaiah A V and Nagaiah K, Eur J Org Chem., 2004, 2004(3), 546-551.

8. $\quad$ Papori G and Babulal D, Tetrahedron Lett., 2009, 50(8), 897-900.

9. Xiongfu Z, Emily S M L, Rosa M A and King LY, Appl Catal A: General, 2004, 261, 109-118.

10. Bhuyan P J and Deb M L, Tetrahedron Lett., 2005, 46, 6453-6456.

11. James D, Lowry M, Jaqua M J and Selepak S T, Applied Microbiology, 1970, 20, 46-53.

12. Barry A L, Procedure for testing antimicrobial agents in agar media: VL Corian (Ed.,) Antibiotics in Lab. Medicine, Williams and Wilkins, Baltimore, MD, 1991, 1-16.

13. Mehta A G and Patel K H, E-J Chem., 2006, 3, 267-273.

14. Barry A L, The Antimicrobial Susceptibility Test Principle and Practices, 1976, 180. 